\title{
Novel Characteristics of Mitochondrial Electron Transport Chain from Eimeria tenella
}

\author{
Makoto Matsubayashi ${ }^{1, *,+}$, Daniel Ken Inaoka ${ }^{2,3,4, *,+} \mathbb{1}$, Keisuke Komatsuya ${ }^{4}$, Takeshi Hatta ${ }^{5}$, \\ Fumiya Kawahara ${ }^{6}$, Kimitoshi Sakamoto ${ }^{7}$, Kenji Hikosaka ${ }^{8}$, Junya Yamagishi ${ }^{9}$, Kazumi Sasai ${ }^{1}$, \\ Tomoo Shiba ${ }^{10}$, Shigeharu Harada ${ }^{10}$, Naotoshi Tsuji ${ }^{5}$ and Kiyoshi Kita ${ }^{2,3,4}$ \\ 1 Division of Veterinary Science, Graduate School of Life and Environmental Sciences, Osaka Prefecture \\ University, 1-58, Rinku Orai Kita, Izumisano, Osaka 598-8531, Japan; ksasai@vet.osakafu-u.ac.jp \\ 2 School of Tropical Medicine and Global Health, Nagasaki University, 1-12-4, Sakamoto, Nagasaki 852-8523, \\ Japan; kitak@nagasaki-u.ac.jp \\ 3 Department of Host-Defense Biochemistry, Institute of Tropical Medicine (NEKKEN), Nagasaki University, \\ 1-12-4, Sakamoto, Nagasaki 852-8523, Japan \\ 4 Department of Biomedical Chemistry, Graduate School of Medicine, The University of Tokyo, 7-3-1, Hongo, \\ Bunkyo-ku, Tokyo 113-0033, Japan; keisuke-kiyose@kcf.biglobe.ne.jp \\ 5 Department of Parasitology, Kitasato University School of Medicine, 1-15-1, Kitasato, Minami-ku, \\ Sagamihara, Kanagawa 252-0374, Japan; htakeshi@med.kitasato-u.ac.jp (T.H.); \\ tsujin@med.kitasato-u.ac.jp (N.T.) \\ 6 Mocky Poultry Practice, Shinmeidai 2-5-33-810, Hamura-shi, Tokyo 205-0023, Japan; funuwo@f7.dion.ne.jp \\ 7 Department of Biochemistry and Molecular Biology, Faculty of Agriculture and Life Science, Hirosaki \\ University, Aomori 036-8561, Japan; sakamok@hirosaki-u.ac.jp \\ 8 Department of Infection and Host Defense, Graduate School of Medicine, Chiba University, 1-8-1, Inohana, \\ Chuo-ku, Chiba 260-8670, Japan; hikosaka@chiba-u.jp \\ 9 Research Center for Zoonosis Control, Hokkaido University, North 20, West 10, Kita-ku, Sapporo, \\ Hokkaido 001-0020, Japan; junya@czc.hokudai.ac.jp \\ 10 Department of Applied Biology, Graduate School of Science Technology, Kyoto Institute of Technology, \\ Matsugasaki, Sakyo-ku, Kyoto 606-8585, Japan; tshiba@kit.ac.jp (T.S.); harada@kit.ac.jp (S.H.) \\ * Correspondence: matsubayashi@vet.osakafu-u.ac.jp (M.M.); danielken@nagasaki-u.ac.jp (D.K.I.); \\ Tel.: +81-72-463-5513 (M.M.); +81-95-819-7230 (D.K.I.) \\ + These authors contributed equally to this work.
}

Received: 28 November 2018; Accepted: 28 December 2018; Published: 8 January 2019

check for updates

\begin{abstract}
Eimeria tenella is an intracellular apicomplexan parasite, which infects cecal epithelial cells from chickens and causes hemorrhagic diarrhea and eventual death. We have previously reported the comparative RNA sequence analysis of the E. tenella sporozoite stage between virulent and precocious strains and showed that the expression of several genes involved in mitochondrial electron transport chain (ETC), such as type II NADH dehydrogenase (NDH-2), complex II (succinate:quinone oxidoreductase), malate:quinone oxidoreductase (MQO), and glycerol-3-phosphate dehydrogenase (G3PDH), were upregulated in virulent strain. To study E. tenella mitochondrial ETC in detail, we developed a reproducible method for preparation of mitochondria-rich fraction from sporozoites, which maintained high specific activities of dehydrogenases, such as NDH-2 followed by G3PDH, MQO, complex II, and dihydroorotate dehydrogenase (DHODH). Of particular importance, we showed that $E$. tenella sporozoite mitochondria possess an intrinsic ability to perform fumarate respiration (via complex II) in addition to the classical oxygen respiration (via complexes III and IV). Further analysis by high-resolution clear native electrophoresis, activity staining, and nano-liquid chromatography tandem-mass spectrometry (nano-LC-MS/MS) provided evidence of a mitochondrial complex II-III-IV supercomplex. Our analysis suggests that complex II from E. tenella has biochemical features distinct to known orthologues and is a potential target for the development of new anticoccidian drugs.
\end{abstract}


Keywords: Coccidium; apicomplexa; Eimeria tenella; membrane protein; mitochondria; electron transport chain; succinate dehydrogenase; ubiquinone; inhibitor

\section{Introduction}

Poultry coccidiosis is an intestinal disease caused by infection of the apicomplexan protozoan parasites from the genus, Eimeria. These parasites produce high economic losses because of decreased chicken growth performance and expensive control systems against the disease. Due to the coccidiosis, the annual worldwide loss in poultry industries, including egg production, was estimated at up to two billion dollars [1]. Amongst the seven Eimeria spp. that infect chicken, Eimeria tenella have been recognized as the most pathogenic parasite due to its high mortality rate following infection. So far, the control of coccidiosis is depending on a variety of anticoccidial agents or live attenuated vaccines. However, these preventive therapies possess severe restricted use in chickens and difficulties to achieve this complete prevention to Eimeria infection. For instant, the routine use of prophylactic medications is not allowed in chicken farms because of the serious problems raised to both the animals' health and chicken product safety. In addition, the administration of live attenuated vaccines to chickens requires re-infection to achieve adequate immunity [2]. Therefore, the development of new cost-effective drugs to carry out satisfactory prevention is currently needed.

In infected chickens, the disruption of the tissue by the Eimeria parasite growth leads to chronic or acute, and watery or bloody diarrhea. The parasites of Eimeria spp. possess a complex life cycle in infected chickens, by initiating the oral ingestion of the sporulated oocysts, including two sporozoites within four sporocysts. The sporozoites released in the intestinal tract invade into the mucosa, and then, undergo several asexual (generating merozoites within schizonts) and sexual stage (generating micro- and macro-gametocytes) developments, resulting in the formation of oocysts [3]. Eventually, these noninfectious oocysts released in the feces begin to sporulate, which is triggered by environmental factors, such as temperature, humidity, and oxygen concentration, to acquire the infectivity [4]. In contrast to the sporulation development outside the host, the asexual and sexual proliferations proceed under low oxygen concentrations in the intestines [5], and thus, it is believed that the Eimeria parasites have evolved specific mechanisms in order to survive and grow within the specialized host microenvironments. However, the molecular basis of the adaptation to the host environment remains unknown.

In intestinal helminth parasites, such as Ascaris suum, Haemonchus contortus, and Echinococcus multilocularis, the mitochondria play key role in adaptation to the host environment by drastically changing the electron transport chain (ETC) composition according to each life cycle stage [6-9]. For instance, the ETC of egg and L3 larvae stages of A. suum have a similar composition to the one from the mammalian host and is composed by respiratory complexes I, II, III, and IV and is dependent on electron carriers, which are ubiquinone and cytochrome $c$, and oxygen as the final electron acceptor by a well-known process named oxygen respiration $[6,10]$. However, because of the low concentration of oxygen in the host intestine, the ETC from the adult stage of A. suum switch to anaerobic respiration known as fumarate respiration, which is composed by complex I coupled with the reverse reaction of complex II and a low potential quinone specie, named rhodoquinone [6]. In fumarate respiration, fumarate is used as the final electron acceptor producing succinate, which is excreted as the end product [11]. In other apicomplexan parasites, such as Plasmodium falciparum or Toxoplasma gondii, which are closely related parasite to Eimeria spp., the parasite's mitochondria are also key organelles involved in cellular metabolic pathways essential to parasite's adaptation and survival within different hosts' environment. Amongst the mitochondrial metabolic pathways, the ETC is believed to be the key pathway for energy generation, pyrimidine de novo biosynthesis, and purine salvage pathways (through fumarate cycle), at least in P. falciparum [12-14]. In addition to ubiquinone, a low potential quinone species involved in anaerobic respiration, menaquinone, has been detected in P. falciparum [15]. 
Due to the indispensability of ETC, it is indeed a promising therapeutic target pathway not only against helminthes, but also protozoan parasites $[6,7,16]$.

In contrast to changes in mitochondria morphology according to the P. falciparum life cycle stages, the mitochondria of E. tenella is observed as elongated structures throughout its life cycle [17]. According to the genome sequence of E. tenella, five ETC dehydrogenase which can utilize oxidized quinone (ubiquinone) as an electron acceptor are conserved: The non-proton motive type II NADH (nicotinamide adenine dinucleotide) dehydrogenase (NDH-2), succinate dehydrogenase (SDH, Complex II), malate:quinone oxidoreductase (MQO), glycerol-3-phosphate (G3P) dehydrogenase (G3PDH), and dihydroorotate (DHO) dehydrogenase (DHODH) [18]. We have previously identified by RNA sequencing analysis that the expression of NDH-2, SDH, MQO, and G3PDH are upregulated in E. tenella at the stages of sporulating and/or excysted sporozoites [19], which deserved a detailed biochemical study. Despite the recognition of these enzymes as potential targets for the development of new chemotherapeutic agents against parasitic diseases, to the best of our knowledge, information about the mitochondria of E. tenella, including the methods for preparation of mitochondria-rich fraction or the activities of the ETC enzymes in detail, are not available.

Here, to determine the biochemical feature of E. tenella ETC enzymes, we have developed a new method for the preparation of mitochondria-rich fraction from E. tenella sporozoites and conducted for the first time, a comprehensive biochemical study of the ETC enzymes.

\section{Materials and Methods}

\subsection{Sequence Analysis}

The sequence analysis of each ETC enzymes deduced from the gene sequence were performed using the ClustalWS platform (https://www.genome.jp/tools-bin/clustalw) and the amino acid percentage identity calculated by the pairwise alignment tool from Jalview 2.9.0b2 software (http: //www.jalview.org/), according to the developer's protocol [20].

\subsection{Preparations of Eimeria tenella Oocysts and Sporozoitess}

The NIAH strain of E. tenella, which was maintained by passage in 2- to 3-week-old chicks (Nisseiken, Tokyo, Japan) at the Laboratory of Parasitic Diseases, National Institute of Animal Health (Tsukuba, Ibaraki, Japan), was used in this study. Chicks were housed in wire-floored cages in coccidian-free rooms and had free access to feed and water that contained no anticoccidial drugs or antibiotics. The animals were treated in accordance with protocols approved by the Animal Care and Use Committee, NIAH (Approval Nos. 11-026, 12-029). Chicks were orally inoculated with $2 \times 10^{4}$ mature oocysts, and feces were collected after 6-8 days. Immature oocysts were purified from feces by using the sugar flotation method [21], incubated for $2-3$ days at $28{ }^{\circ} \mathrm{C}$ in $2.5 \%(\mathrm{w} / \mathrm{v})$ potassium dichromate (Wako, Osaka, Japan) to promote sporulation, and then stored at $4{ }^{\circ} \mathrm{C}$ for $2-3$ months before use.

For purification of sporozoites, sporulated oocysts $\left(3 \times 10^{8}\right)$ were centrifuged at $1500 \times g$ for $5 \mathrm{~min}$ at $4{ }^{\circ} \mathrm{C}$ and washed with phosphate-buffered saline (PBS). The oocysts were further incubated in $8.5 \%-13.5 \%(\mathrm{w} / \mathrm{v})$ sodium hypochlorite for $20 \mathrm{~min}$ at $4{ }^{\circ} \mathrm{C}$ and centrifuged at $1500 \times g$ for $5 \mathrm{~min}$ at $4{ }^{\circ} \mathrm{C}$. The sodium hypochlorite treatment was repeated twice, followed by washing with PBS for three times. After the last centrifugation, parasites were incubated in $4 \mathrm{~mL}$ of excystation medium $[0.25 \%$ $(\mathrm{w} / \mathrm{v})$ trypsin (Merck, Darmstadt, Germany) and 1\% (w/v) taurodeoxycholic acid (Sigma, St. Louis, MO, USA) in Hanks' balanced salt solution (Sigma), pH 7.4], at $4{ }^{\circ} \mathrm{C}$ for overnight. Oocysts were centrifuged at $1750 \times \mathrm{g}$ for $10 \mathrm{~min}$ at $4{ }^{\circ} \mathrm{C}$, suspended in $1 \mathrm{~mL}$ of PBS. Next, $1 \mathrm{~mL}$ of $0.5-0.7 \mathrm{~mm}$ glass beads (As one, Osaka, Japan) was added and the oocysts were broken by vortexing for approximately $2.5 \mathrm{~min}$ at room temperature until $>95 \%$ of the oocysts were broken, releasing the sporocysts, under a microscopic observation. Ten milliliters of PBS were added, mixed briefly, and the parasites were collected by retrieving the supernatant. This step was repeated four times. Collected parasites were 
centrifuged at $1400 \times g$ for $10 \mathrm{~min}$ at $4{ }^{\circ} \mathrm{C}$, re-suspended in $20 \mathrm{~mL}$ of excystation medium, and incubated in a water bath shaker for $2 \mathrm{~h}$ at $41^{\circ} \mathrm{C}$ with vigorous shaking of $160 \mathrm{rpm}$. After adding PBS up to $50 \mathrm{~mL}$, parasites, including excysted sporozoites, were collected by centrifugation at $1750 \times g$ for $10 \mathrm{~min}$ at room temperature. Next, the pellet was washed once in $50 \mathrm{~mL}$ of PBS. After centrifugation at $1750 \times g, 10 \mathrm{~min}$ at room temperature, the parasites were suspended in $20 \mathrm{~mL}$ PBS and filtered by a passage in a membrane filter of $20 \mu \mathrm{m}$ (Steriflip, Merck Millipore, Darmstadt, Germany). The filter was washed by an additional $80 \mathrm{~mL}$ of PBS, and the parasites were concentrated by centrifugation at $1750 \times g, 10 \mathrm{~min}$ at room temperature. The parasites were suspended in $25 \mathrm{~mL}$ of PBS and centrifuged at $100 \times g$ for 2 min at $4{ }^{\circ} \mathrm{C}$. The supernatant, containing the sporozoites, was collected and kept on ice. The remaining pellet was resuspended in $25 \mathrm{~mL}$ of PBS centrifuged again at $100 \times \mathrm{g}$ for $2 \mathrm{~min}$ at $4{ }^{\circ} \mathrm{C}$. The supernatant was collected and combined to the supernatant from the previous step. A total of $50 \mathrm{~mL}$ of combined supernatant was centrifuged at $1800 \times \mathrm{g}$ for $10 \mathrm{~min}$ twice, and the pellets consisting of sporozoites (approximately $3 \times 10^{8}$ ) were resuspended in $12.5 \mathrm{~mL}$ of MSE buffer ( $210 \mathrm{mM}$ mannitol, $10 \mathrm{mM}$ sucrose, $1 \mathrm{mM}$ disodium EDTA (ethylenediaminetetraacetic acid), and $50 \mathrm{mM}$ Tris- $\mathrm{HCl}, \mathrm{pH}$ 7.5) containing $10 \mathrm{mM}$ disodium malonate and protease inhibitor cocktail (Roche Diagnostics, Tokyo, Japan). The parasite suspension was centrifuged at $1800 \times g$ for $10 \mathrm{~min}$ at $4{ }^{\circ} \mathrm{C}$. The final pellet was suspended in $5 \mathrm{~mL}$ of MSE buffer.

\subsection{Transmission Electron Microscopy (TEM) of Eimeria tenella}

To morphologically confirm the mitochondria of E. tenella, the sporozoites prepared as described above were fixed with $2 \%(\mathrm{w} / \mathrm{v})$ glutaraldehyde (Electron Microscopy Sciences, Hatfield, PA, USA) and $2 \%(\mathrm{w} / \mathrm{v})$ paraformaldehyde (PFA, TAAB laboratories Equipment, Berks, UK) in $0.1 \mathrm{M}$ phosphate buffer (PB, pH 7.4) at room temperature for $2 \mathrm{~h}$ and kept at $4{ }^{\circ} \mathrm{C}$ overnight. The cells were washed with $0.1 \mathrm{M} \mathrm{PB}$ (pH 7.4) containing $0.1 \mathrm{M}$ sucrose (Wako, Osaka, Japan), post-fixed with $1 \%$ (w/v) OsO4 in $0.1 \mathrm{M} \mathrm{PB}$ containing $0.1 \mathrm{M}$ sucrose at $4{ }^{\circ} \mathrm{C}$ for $2 \mathrm{~h}$, dehydrated in an ethanol series, and embedded in Epon resin (TAAB Laboratories Equipment). Ultra-thin sections were stained with saturated uranyl acetate (TAAB Laboratories Equipment) in distilled water for 20 min and Reynold's lead citrate for 3 min and examined with an electron microscope (H-7500, Hitachi, Tokyo, Japan).

\subsection{Preparation of Eimeria tenella Mitochondria-rich Fraction}

The mitochondria-rich fraction of E. tenella sporozoites were obtained by two different cell disruption protocols. The purified sporozoites were disrupted using (i) the POLYTRON homogenizer (POLYTRON ${ }^{\circledR}$ PT1200E, Central Scientific Commerce, Tokyo, Japan) at 70\% of the maximum output by a cycle consisting of 2 min disruption and 5 min on ice for two cycles; or (ii) $\mathrm{N}_{2}$ cavitation (4639 Cell disruption Bomb, Parr, Moline, IL, USA) at 2000 psi for $20 \mathrm{~min}$ at $4{ }^{\circ} \mathrm{C}$. Unbroken cells and cell debris were removed by centrifugation at $700 \times g$ for $10 \mathrm{~min}$ at $4{ }^{\circ} \mathrm{C}$, and the supernatant was centrifuged at $30,000 \times \mathrm{g}$ for $30 \mathrm{~min}$ at $4{ }^{\circ} \mathrm{C}$. The mitochondria-rich fraction was recovered as the precipitate, which was suspended in MSE containing $10 \mathrm{mM}$ disodium malonate and protease inhibitor cocktail. The protein concentration was determined according to the method of Lowry using bovine serum albumin as a standard [22].

\subsection{Enzyme Assays}

All enzyme assays were performed using $5-10 \mu \mathrm{g}$ of the mitochondria-rich fraction using black quartz cuvettes in $0.6 \mathrm{~mL}$ reaction mixture at $25^{\circ} \mathrm{C}$. The reagents used in each assay were mixed with a reaction buffer containing $50 \mathrm{mM}$ potassium phosphate ( $\mathrm{pH}$ 7.4) (KPi Buffer) and 2 mM EDTA.

The NADH oxidase activity (NDH-2-Complex III-IV) was assay by the addition of $0.2 \mathrm{mM}$ NADH to the mixture and following the decrease in absorbance of NADH at $340 \mathrm{~nm}\left(\varepsilon=6.2 \mathrm{mM}^{-1} \mathrm{~cm}^{-1}\right)$ with UV-3000 double wavelength spectrophotometer (Shimadzu Corp., Kyoto, Japan).

Cytochrome $c$-linked NADH, G3P, malate, succinate, and DHO dehydrogenase activities were determined in a reaction mixture containing $30 \mu \mathrm{M}$ horse heart cytochrome $c$ and $2 \mathrm{mM} \mathrm{KCN}$ at 
$550 \mathrm{~nm}\left(\varepsilon=19 \mathrm{mM}^{-1} \mathrm{~cm}^{-1}\right)$ by adding $0.2 \mathrm{mM} \mathrm{NADH}, 5 \mathrm{mM}$ malate, $5 \mathrm{mM} \mathrm{G} 3 \mathrm{P}, 8.3 \mathrm{mM}$ succinate, and $0.4 \mathrm{mM} \mathrm{DHO}$, respectively, into the reaction buffer.

Individual G3PDH, MQO, SDH, and DHODH activities were assayed at $278 \mathrm{~nm}$ in the presence of each substrate as described above, however, instead of cytochrome $c, 50 \mu \mathrm{M}$ of decylubiquinone (dUQ) $\left(\varepsilon=15 \mathrm{mM}^{-1} \mathrm{~cm}^{-1}\right)$ was added. The NDH-2 activity was measured following the consumption of NADH at $340 \mathrm{~nm}$.

\subsection{Analysis of the Quinone from Eimeria tenella}

Quinone contents were analyzed by liquid chromatography-mass spectrometry (LC-MS) and the high-performance liquid chromatography equipped with photodiode array detector (HPLC-PDA) systems. To the $30 \mathrm{mg}$ wet pellet of the E. tenella sporozoites, $600 \mu \mathrm{L}$ of 2-propanol was added to extract quinones and quinols. To the $600 \mu \mathrm{L}$ of supernatant, $6 \mu \mathrm{L}$ of 2-propanol supplemented with $120 \mathrm{mM} \mathrm{FeCl}_{3}$ was added to oxidize all the quinol to the quinone [23]. After the removal of insoluble debris by filtration through a $0.2 \mu \mathrm{m}$ pore-size filter (Ultra-free, millipore), $4 \mu \mathrm{L}$ of the filtrated extracts was injected to the LC-MS system, consisting of a pump (L-2100, Hitachi, Japan), column oven at $40{ }^{\circ} \mathrm{C}$ (L-2300, Hitachi), analytical column (Inertsil ODS-3, $2 \mu \mathrm{m}, 2.1 \mathrm{~mm}$ i.d. $\times 50 \mathrm{~mm}$, GL Science, Tokyo, Japan), UV detector at $275 \mathrm{~nm}$ (L-2420, Hitachi), and ESI-TOF MS (NanoFrontier LD, Hitachi). The mobile phase used in our experiment was methanol/2-propanol/water $(45 / 48 / 7, \mathrm{v} / \mathrm{v} / \mathrm{v}), 0.1 \%$ $(\mathrm{v} / \mathrm{v})$ formic acid at a flow rate of $0.2 \mathrm{~mL} / \mathrm{min}$. To analyze with the HPLC-PDA system, $600 \mu \mathrm{L}$ of oxidized extract was dried under nitrogen gas flow. The concentrated solution was obtained by adding $30 \mu \mathrm{L}$ of 2-propanol and filtrated as described above. The HPLC-PDA system consisting of a pump (L-7610, Hitachi), column oven at $40{ }^{\circ} \mathrm{C}$ (L-7300, Hitachi), analytical column (Inertsil ODS-3, $2 \mu \mathrm{m}$, $2.1 \mathrm{~mm}$ i.d. $\times 50 \mathrm{~mm}$, GL Science), and PDA detector (MD-4017, Jasco, Tokyo, Japan) was operated under the same mobile phase as the LC-MS analysis. As standard samples, menaquinone-7 (MK-7) (Wako, Osaka, Japan), ubiquinone-9 (UQ-9) (Sigma, St. Louis, MO, USA), and UQ-10 (Wako) were used. For UQ-8, MK-8, and demethylmenaquinone-8 (DMK-8), Escherichia coli HB101 extract was prepared essentially following the procedure described above for E. tenella quinone extraction.

\subsection{Enzyme Inhibition Assay of Eimeria tenella Succinate Dehydrogenase}

The $50 \%$ inhibitory concentration $\left(\mathrm{IC}_{50}\right)$ values against succinate-cytochrome $c$ reductase activity (Complex II-III) of the parasite mitochondria was determined varying the concentration of four compounds, which included atpenin A5 and carboxin, which are known as inhibitors for bovine complex II with the $\mathrm{IC}_{50}$ values of 0.004 and $1 \mu \mathrm{M}$, respectively [24]; siccanin, which was reported as an inhibitor of complex II from Leishmania tarentolae, L. donovani, Trypanosoma brucei, and T. cruzi with $\mathrm{IC}_{50}$ value of $0.190 \mu \mathrm{M}, 1.17 \mu \mathrm{M}, 0.368 \mu \mathrm{M}$, and $1.48 \mu \mathrm{M}$, respectively [25,26]; and flutolanil, which was reported as a potent and specific inhibitor of Ascaris suum complex II with an $\mathrm{IC}_{50}$ value of $0.081 \mu \mathrm{M}$ [27]. As the positive control for complex II-III inhibition assay, $0.1 \mu \mathrm{M}$ of stigmatellin (complex III inhibitor) was used [28].

\subsection{High Resolution Clear Native Electrophoresis ( $\mathrm{HrCNE}$ ) and Succinate Dehydrogenase Activity Staining}

The mitochondria-rich fraction of E. tenella sporozoites prepared as described above were incubated on ice for $10 \mathrm{~min}$ in $50 \mathrm{mM}$ Tris- $\mathrm{HCl} \mathrm{pH} \mathrm{8.0,10 \%} \mathrm{(v/v)} \mathrm{glycerol} \mathrm{and} 0.5 \%(\mathrm{w} / \mathrm{v})$ sucrose monolaurate (SML). The solubilized mitochondrial membrane proteins were centrifuged at $200,000 \times g$ for $15 \mathrm{~min}$ at $4{ }^{\circ} \mathrm{C}$. The resulting supernatant was mixed with final concentrations of $0.01 \%(\mathrm{w} / \mathrm{v})$ Ponceau S in $10 \mathrm{mM}$ Tris-HCl, pH 7.5 (Invitrogen, Carlsbad, CA, USA), 5\% (v/v) glycerol, and loaded onto Novex $4 \%-16 \%(\mathrm{w} / \mathrm{v})$ Bis-Tris gels (Invitrogen). HrCNE was performed in NativePAGE ${ }^{\mathrm{TM}}$ running buffer (Invitrogen) supplemented with $0.02 \%(\mathrm{w} / \mathrm{v})$ dodecylmaltoside and $0.05 \%(\mathrm{w} / \mathrm{v})$ sodium deoxycholate at $150 \mathrm{~V}$ for $1 \mathrm{~h}$, and then increased to $250 \mathrm{~V}$ under voltage constant at $4{ }^{\circ} \mathrm{C}$. After the HrCNE, the gels were stained either by Coomassie brilliant blue (CBB) dye or subjected to SDH activity staining. 
The SDH activity staining of mitochondrial ETC proteins was conducted by the modified methods as in a previous report [26]. After the $\mathrm{HrCNE}$, the gels were washed twice in $5 \mathrm{mM}$ Tris- $\mathrm{HCl}$ buffer ( $\mathrm{pH}$ 7.4) for $5 \mathrm{~min}$ to remove the detergents from the running buffer. The equilibrated gel was soaked in $5 \mathrm{mM}$ Tris- $\mathrm{HCl}$ buffer ( $\mathrm{pH}$ 7.4) containing $2.5 \mathrm{mg} / \mathrm{mL}$ nitro blue tetrazolium chloride at room temperature for $5 \mathrm{~min}$. Next, succinate and phenazine methosulfate were added at a final concentration of $20 \mathrm{mM}$ and $4 \mathrm{mg} / \mathrm{mL}$, respectively, and kept static in the dark. After the SDH stained band was clearly visible, the reaction was stopped by washing the gel several times with pure water.

2.9. Two Dimentional Sodium Dodecyl Sulfate Polyacrylamide Gel Electrophoresis (2D-SDS-PAGE) and NanoLiquid Chromatography Tandem-Mass Spectrometry (LC MS/MS) Analysis of Eimeria tenella Succinate Dehydrogenase Stained Band

The SDH activity staining band was cut out from the HrCNE gels, soaked in $30 \mu \mathrm{L}$ of $1 \times$ SDS-PAGE sample buffer for $5 \mathrm{~min}$, and loaded onto $12.5 \%(\mathrm{w} / \mathrm{v})$ SDS-PAGE gel. The gels were stained by CBB for the visualization of proteins composing the SDH stained band. Additionally, the SDH activity stained band was sent for analysis by nano-LC-MS/MS (FUJIFILM Wako Pure Chemical Corporation). Identified protein fragment sequences were searched in the E. tenella genome database (http:/ / protists.ensembl.org) using the MASCOT software (Matrix Science, Tokyo, Japan).

\section{Results}

\subsection{Electron Transport Chain Dehydrogenases from Eimeria tenella}

Five ETC dehydrogenases are conserved in the genome of E. tenella, which are the NDH-2, Complex II, MQO, G3PDH, and DHODH (Figures S1-S5). NDH-2 (Figure S1) is an enzyme with activity equivalent to respiratory complex $\mathrm{I}$, but devoid of proton-pump activity and is required to re-oxidize NADH generated by mitochondrial pathways, such as the tricarboxylic acid (TCA) cycle [29]. Complex II is the only membrane bound enzyme from the TCA cycle in mammals and catalyzes the electron transfer from succinate to quinone (SQR) or from reduced quinol to fumarate (QFR) $[7,24,26,30]$. In mammals, complex II is composed by flavoprotein (SDH1, Figure S2), iron-sulfur cluster protein (SDH2, Figure S3), cytochrome $b$ large (SDH3), and small subunits (SDH4). The SDH1 and SDH2 are well conserved in prokaryotes and eukaryotes (Figures S2 and S3) while the membrane anchor subunits formed by SDH3 and SDH4 are diverse [6]. MQO is a membrane protein found among bacteria kingdom and some protozoa (Figure S4) and functions as a member of the TCA cycle. Higher eukaryotes do not possess $\mathrm{MQO}$, but instead, they possess the $\mathrm{NAD}^{+}$-dependent malate dehydrogenase, a soluble enzyme located in the mitochondria matrix $[13,14,31]$. G3PDH catalyzes the irreversible oxidation of G3P into dihydroxyacetone phosphate using ubiquinone as the electron acceptor and is well conserved among prokaryotes and eukaryotes (Figure S5). It is an enzyme involved in glycerol metabolism as well as gluconeogenesis [32,33]. Finally, DHODH catalyzes the oxidation of dihydroorotate into orotate with concomitant reduction of ubiquinone [34]. DHODH is the rate-limiting step in the pyrimidine de novo biosynthesis pathway and is required to generate UMP, the building block of DNA and RNA necessary to sustain life [35,36]. NDH-2 and MQO are not conserved in the animal kingdom and are considered as potential drug targets for the development of new antibiotics, and antifungal and antiparasitic agents [13,14,29].

\subsection{Transmission Electron Microscopy Analysis of Eimeria tenella}

The mitochondria within the sporozoites were morphologically confirmed by TEM. As shown in Figure $1 \mathrm{a}, \mathrm{b}$, the mitochondria from E. tenella possessing tubular cristae were observed around the anterior and posterior refractile bodies. In addition, the mitochondria from sporozoites could be stained by MitoTracker ${ }^{\circledR}$ (Thermo Fisher Scientific, Waltham, MA, USA) (Figure 1c) and were observed surrounding the entire refractile body, which was consistent with the TEM analysis. 


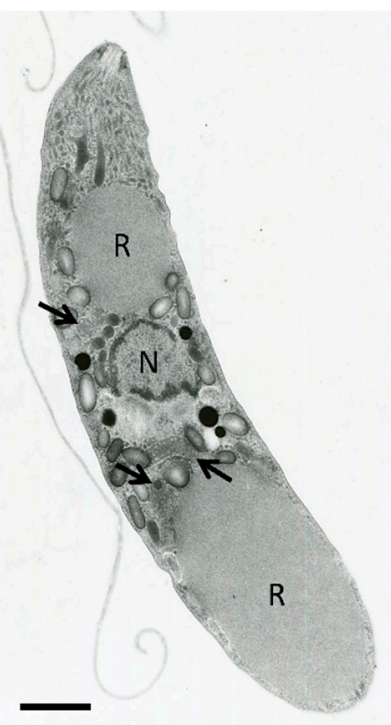

(a)

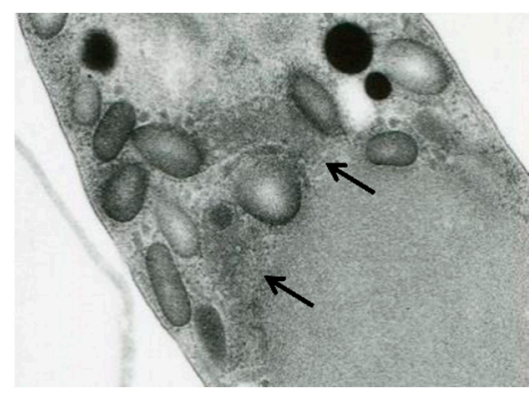

(b)

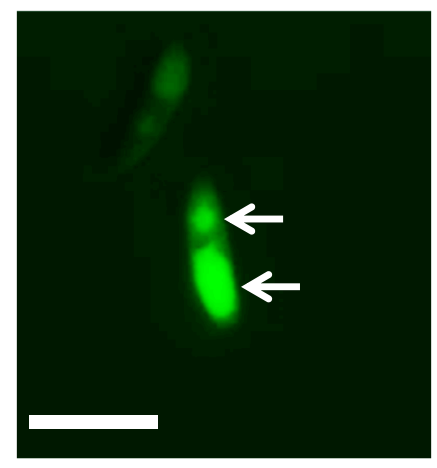

(c)

Figure 1. (a) Electron microscopy of Eimeria tenella sporozoite; (b) high magnification of the mitochondria of the parasite; (c) staining of sporozoites by mitochondrial specific probes (MitoTracker ${ }^{\circledR}$, Thermo Fisher Scientific). Bars $=2 \mu \mathrm{m}$ (a) and $5 \mu \mathrm{m}$ (c). The positions of the mitochondria are indicated by the arrows. R: refractile bodies. N: nucleous.

\subsection{Preparation of Mitochondria-Rich Fraction}

To perform biochemical study of E. tenella mitochondrial ETC enzymes, we evaluated the efficiency of the methods for preparation of mitochondria-rich fraction by two different cell disruptions protocols (Polytron or $\mathrm{N}_{2}$ cavitation) from E. tenella sporozoites. Also, the storage conditions that maintain stable mitochondrial NADH-cyt $c$ and succinate-cyt $c$ activities. As a result, the mitochondria-rich fraction obtained from sporozoites disrupted by the Polytron method exhibited a lower total activity for both activities than those disrupted by the $\mathrm{N}_{2}$ cavitation method (Table 1). When the mitochondria-rich fraction was stored at $4{ }^{\circ} \mathrm{C}$ for $48 \mathrm{~h}$, both activities were reduced independently to the disruption method. However, when the mitochondria-rich fraction stored at $-80^{\circ} \mathrm{C}$ for $48 \mathrm{~h}$, the NADH-cyt $c$ and succinate-cyt $c$ activities remained at $100 \%$ and $65 \%$ for the Polytron method and $74 \%$ and $87 \%$ for the $\mathrm{N}_{2}$ cavitation method, respectively. Therefore, we determined the specific activity of E. tenella ETC enzymes on the same day as the mitochondria-rich fraction isolation. For inhibition studies, the mitochondria-rich fraction stored at $-80^{\circ} \mathrm{C}$ was used within 3-4 days due to the higher yield of both activities compared to the Polytron method. By this protocol, we could obtain approximately $1.0-1.5 \mathrm{mg}$ of mitochondria-rich fraction starting from $3 \times 10^{8}$ sporozoites.

Table 1. Stability of NADH-cyt $c$ and succinate-cyt $c$ activities (Abs $/ \mathrm{min} / \mu \mathrm{L})$.

\begin{tabular}{ccc}
\hline & NADH-cyt $c$ & Succinate-cyt $c$ \\
\hline Day 0-Polytron & $0.011(100 \%)$ & $0.004(100 \%)$ \\
Day 0-N $\mathrm{N}_{2}$ Cavitation & $0.050(100 \%)$ & $0.010(100 \%)$ \\
Day 3-4 ${ }^{\circ} \mathrm{C}-$ Polytron & $0.006(57 \%)$ & $0.003(63 \%)$ \\
Day 3-4 ${ }^{\circ} \mathrm{C}-\mathrm{N}_{2}$ Cavitation & $0.008(16 \%)$ & $0.007(69 \%)$ \\
Day 3-80 ${ }^{\circ} \mathrm{C}-$ Polytron & $0.011(100 \%)$ & $0.026(65 \%)$ \\
Day 3-80 ${ }^{\circ} \mathrm{C}-\mathrm{N}_{2}$ Cavitation & $0.037(74 \%)$ & $0.009(87 \%)$ \\
\hline
\end{tabular}

Data from E. tenella mitochondria prepared by two different methods (Polytron or $\mathrm{N}_{2}$ cavitation), at day 0 and day 3 stored at $4{ }^{\circ} \mathrm{C}$ or $-80^{\circ} \mathrm{C}$. The number in parenthesis represents the percentage activity relative to day 0 . NADH: nicotinamide adenine dinucleotide. 


\subsection{Characterization of Eimeria tenella Mitochondrial Electron Transport Chain.}

Next, the specific enzyme activities of the mitochondrial respiratory chain from E. tenella sporozoites were determined and are summarized in Table 2. The NADH-oxidase specific activity of $5.9 \mathrm{nmol} / \mathrm{min} / \mathrm{mg}$ protein was confirmed in the mitochondria-rich fraction from E. tenella sporozoites, which was completely inhibited by the addition of $2 \mathrm{mM} \mathrm{KCN}$, an inhibitor of complex IV. Consecutive addition of fumarate restored the NADH oxidation activity to $1.3 \mathrm{nmol} / \mathrm{min} / \mathrm{mg}$ protein, and following the addition of $5 \mathrm{mM}$ malonate, this activity was again abolished. This indicates that E. tenella possesses an intrinsic capacity to re-route the ETC dehydrogenase electron flux from oxygen to fumarate via reverse reaction of complex II, when the electron flux to oxygen is compromised. Consistently, by LC-MS (m/z 741.56 [M + Na $]^{+}$) and HPLC-PDA analyses, we could identify a menaquinone specie, MK-8 $\left(\mathrm{H}_{2}\right)$, from the extracts of E. tenella sporozoites (Figures S6 and S7). Menaquinones are usually required for anaerobic respiration, such as fumarate respiration, in bacteria living under anaerobic conditions [37]. Moreover, all ETC enzyme activity deduced from the E. tenella genome could be determined (Table 2) either by cytochrome $c$-linked (via complex III) or by direct detection of dUQ reduction (Table 3). Amongst the five ETC dehydrogenases, NDH-2 showed the highest activity, followed by G3PDH/MQO, SDH, and DHODH (Table 3).

Table 2. Reproducibility of mitochondria preparation from E. tenella sporozoites.

\begin{tabular}{ccccc}
\hline & \multicolumn{4}{c}{ Specific Activity (nmol/min/mg) } \\
\cline { 2 - 5 } & A & B & C & D \\
\hline NADH-cyt $c^{1}$ & $341 \pm 18$ & $228 \pm 16$ & $167 \pm 8$ & $189 \pm 28$ \\
G3P-cyt $c^{1}$ & $137 \pm 3$ & $94 \pm 1$ & $101 \pm 6$ & $119 \pm 5$ \\
Malate-cyt $c^{1}$ & $108 \pm 9$ & $75 \pm 9$ & $95 \pm 5$ & $77 \pm 9$ \\
Succinate-cyt $c^{1}$ & $50 \pm 7$ & $50 \pm 7$ & $71 \pm 2$ & $54 \pm 2$ \\
Dihydroorotate-cyt $c^{1}$ & 0.0 & 0.0 & 1.7 & 1.7 \\
\hline
\end{tabular}

1 Data represent the average of cytochrome $c$-linked specific activity measured in triplicates, except for dihydroorotate-cyt $c$ activity, by four independent experiment (A, B, C, and D) performed at different dates.

Table 3. Specific activities of E. tenella electron transport chain dehydrogenases.

\begin{tabular}{lc}
\hline & Specific Activity $^{\mathbf{1}}$ \\
\hline NADH oxidase & $5.9 \pm 0.9$ \\
$+2 \mathrm{mM} \mathrm{KCN}$ & $0.0 \pm 0.3$ \\
+ 2 mM fumarate & $1.3 \pm 0.3$ \\
+ 5 mM malonate & $-0.5 \pm 0.3$ \\
NADH-cyt $c$ & $167 \pm 8$ \\
NADH-dUQ & $224 \pm 19$ \\
\hline G3P-cyt $c$ & $101 \pm 6$ \\
G3P-dUQ & $61 \pm 2$ \\
\hline Malate-cyt $c$ & $95 \pm 5$ \\
Malate-dUQ & $67 \pm 3$ \\
\hline Succinate-cyt $c$ & $71 \pm 2$ \\
Succinate-dUQ & $25 \pm 2$ \\
\hline Dihydroorotate-cyt $c$ & 1.7 \\
\hline
\end{tabular}

${ }^{1}$ Data represent the average of specific activity $(\mu \mathrm{mol} / \mathrm{min} / \mathrm{mg}$ protein) measured in triplicates from experiment $\mathrm{C}$ (Table 2), except for dihydroorotate-cyt $c$ activity.

\subsection{Inhibition Studies of Eimeria tenella Mitochondrial Complex II}

We have previous reported that the sensitivity of complex II to classical inhibitors can vary between species [25-27,38]. To evaluate the sensitivity of E. tenella complex II to inhibitors, we tested the inhibitory effect of classical complex II inhibitors, such as atpenin A5, siccanin, carboxin, and 
flutolanil. Surprisingly, none of the compounds tested showed significant inhibition of complex II-III activity, except for siccanin, which showed an $\mathrm{IC}_{50}$ of $4.0 \mu \mathrm{M}$ (Table 4). Siccanin was previously identified as inhibitor of complex II from fungi, Pseudomonas aeruginosa, rat, L. tarentolae, L. major, Trypanosoma brucei, and T. cruzi $[25,26]$. Although siccanin inhibited E. tenella complex II-III activity, it did not inhibit the SQR activity of complex II (Table 4), indicating that the primary target of siccanin in E. tenella is complex III rather than complex II.

Table 4. Insensitivity of E. tenella complex II to classical inhibitors.

\begin{tabular}{ccccc}
\hline & \multicolumn{4}{c}{ IC $_{\mathbf{5 0}}$ of Succinate-cyt $\boldsymbol{c}$ Activity $(\boldsymbol{\mu M})$} \\
\cline { 2 - 5 } & E. tenella & $\boldsymbol{P .}$ y. yoelii [39] & Rat & Porcine \\
\hline Atpenin A5 & $>50$ & $4.2 \pm 0.2[39]$ & $0.007 \pm 0.0003[39]$ & N.D. \\
Siccanin & $4.0 \pm 0.6(>50)^{1}$ & N.D. [39] & $9.3 \pm 1.0[25]$ & $861 \pm 822[25]$ \\
Carboxin & $>50$ & $3.6 \pm 1.0[39]$ & $3.8 \pm 0.1[39]$ & N.D. \\
Flutolanil & $>50$ & $>100[39]$ & $>100[39]$ & $>50[7]$ \\
Stigmatellin & $78.4 \pm 13.1 \%{ }^{2}$ & N.D. & N.D. & N.D. \\
\hline
\end{tabular}

${ }^{1}$ Value in parenthesis is the $\mathrm{IC}_{50}$ determined for succinate-dUQ activity. ${ }^{2}$ Percentage inhibition at $100 \mathrm{nM}$ concentration of stigmatellin, which was used as a positive control for complex III inhibition. N.D.: Not determined.

\subsection{HrCNE and Nano-LC MS/MS Analyses of Eimeria tenella Mitochondrial Complex II}

Due to the unusual property of E. tenella complex II, we further conducted analysis by HrCNE. As shown in Figure 2, the HrCNE followed by SDH activity staining revealed a single band corresponding to the molecular weight of $745 \mathrm{kDa}$. As a control, bovine mitochondria was included and showed a band of about $240 \mathrm{kDa}$, which was consistent to a previous report (Figure 2) [26]. Moreover, the band corresponding to E. tenella complex II was excised from HrCNE gel and loaded onto 2-D SDS-PAGE. According to the result shown in Figure 2, 15 bands (SDH17 to SDH150, with numbers indicating the calculated molecular weight), stoichiometric stained by CBB, were identified from the SDH activity stained band. Additionally, the band corresponding to SDH from HrCNE was also sent for nano-LC-MS/MS analysis. Among the SDH subunits, we could successfully identify the Fp (flavoprotein) and a possible candidate for the Ip (iron-sulfur protein) subunits in the SDH activity stained band (Table S1). In addition, several subunits from complex III (ubiquinol:cytochrome $c$ reductase), as well as complex IV (Cytochrome $c$ oxidase) and cytochrome $c$ itself, were identified in the SDH activity stained band (Table S1).

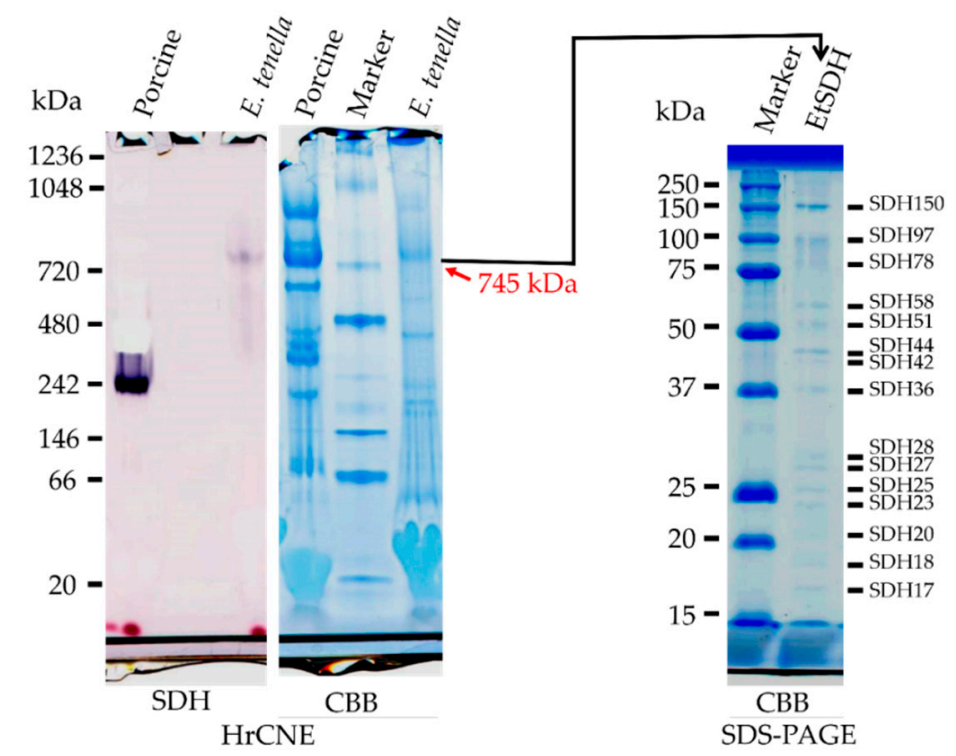

Figure 2. High Resolution Clear Native Electrophoresis (HrCNE) (left and middle) and two-dimensional polyacrylamide gel electrophoresis (2D-SDS-PAGE) (right) analysis of E. tenella complex II. For HrCNE 
gels, $10 \mu \mathrm{g}$ and $27 \mu \mathrm{g}$ per lane of bovine heart mitochondria and E. tenella mitochondria-rich fraction were loaded, respectively. Left and middle panels were stained by SDH activity and CBB staining, respectively. The red arrow indicates the location of $\mathrm{SDH}$ stained bands of $745 \mathrm{kDa}$ in the CBB stained gel, which was cut off and loaded into 2D-SDS-PAGE gel (right) and stained by CBB. A total of 15 bands, named according to their calculated molecular weight, were stoichiometrically stained by CBB (right).

\section{Discussion}

This study provides a reproducible method for the preparation of mitochondria-rich fraction from E. tenella sporozoites. Using this method, a high specific activity of ETC enzymes was observed in our preparations and characterized for the first time. According to our TEM analysis, the tubular mitochondria from sporozoites contains tubular cristae and is localized in the vicinity of the refractile body. Since the curvature of the cristae membrane is driven by the adenosine triphosphate (ATP) synthase [40], our result indicates the presence of an active ETC and oxidative phosphorylation, which is consistent with the high ETC activity observed in our study. In the case of the P. falciparum and $P$. berghei asexual blood stage, the cristae are not found in their mitochondria, whilst in the gametocytes, the presence of cristae becomes apparent [41,42]. Interestingly, the MitoTracker ${ }^{\circledR}$ (Thermo Fisher Scientific) stained mitochondria were observed in close vicinity to the refractile body. The physiological function of the refractile body is still unclear, but it has been found in Eimeria and Lankesterella [43]. Because of the close location of mitochondria to the refractile body in E. tenella sporozoites, it is tempting to speculate that the overlapping spatial distribution of mitochondria and the refractive body might be important for their respective functions.

Previously, a cell disruption method using Teflon pestle tissue homogenizer was reported [44]. However, due to the in situ heating causing a loss of ETC enzyme activities, the longer time needed, and problems with reproducibility, this method was not tested in this study. Instead, we tested the Polytron homogenizer, which is a mild cell disruption method. We have previously reported a fast and successful disruption of P. falciparum and P. berghei by the $\mathrm{N}_{2}$ cavitation method [45-47], and in this study, we have compared the two methods in terms of the yield of ETC enzyme activity (NADH- and succinate-cytochrome $c$ reductase) in a mitochondria-rich fraction obtained from E. tenella sporozoites. Although Plasmodium parasites at the erythrocytic stage could be disrupted by $\mathrm{N}_{2}$ cavitation at 1200 psi, E. tenella sporozoites could not be disrupted at the same condition, but it required a higher pressure of 2000 psi. This high pressure might be needed to break the specific membrane structure of the sporozoites due to its ability to survive in the harsh environment of the chick intestine, where the parasites are exposed to digestive enzymes. At least for NADH- and succinate-cytochrome $c$ reductase activities, we have found that storage of mitochondria-rich fraction at $-80{ }^{\circ} \mathrm{C}$ maintained the specific activity by $74 \%$ and $87 \%$, respectively, at least for three days of storage.

The enrichment of mitochondria is evident due to the extremely high specific activities of NDH-2, and Complex II and III activities in our preparations. The NADH-cytochrome $c$ reductase activity from our preparations ranged from 167 to $341 \mathrm{nmol} / \mathrm{min} / \mathrm{mg}$, which is over 10-fold higher than the reported Plasmodium mitochondria-rich preparations [39]. Similarly, complex II [41,48,49] and MQO [14] specific activities from our preparations exceeded the reported ones from Plasmodium. Although the specific activities of ETC enzymes indicate an enrichment of the mitochondria fraction, microneme and rhoptory proteins were detected by nano LC-MS/MS analysis in the SDH activity stained band by HrCNE (Table S1). This suggests that those organelles are present in the mitochondria-rich fraction used in this study.

The biochemical features of the mitochondrial energy metabolic pathway related to ETC in E. tenella has not been fully clarified. Previously, the mRNA expressions for most enzymes in the metabolic pathway, including glycolysis and the TCA cycle, have been reported at the extracellular stages of E. tenella [19], and mitochondria was confirmed in the E. tenella sporozoites with tubular cristae [50]. Biochemical analysis of the mitochondria-rich fraction revealed that the E. tenella sporozoites have an 
active ETC, characterized by high NDH-2 activity, followed by MQO, G3PDH, SDH, and DHODH activities. The presence of $\mathrm{NDH}-2, \mathrm{SDH}$, and $\mathrm{MQO}$ indicates that at the sporozoite stage, the parasites possess an active TCA cycle. In addition, G3PDH activity indicates the presence of an active glycerol metabolism and perhaps gluconeogenesis. In contrast to other ETC dehydrogenases, the DHODH activity in sporozoites was found to be the lowest $(0-1.7 \mathrm{nmol} / \mathrm{min} / \mathrm{mg})$. Since DHODH is the rate-limiting step in pyrimidine de novo biosynthesis required for DNA and RNA synthesis, low DHODH activity is characteristic of non-dividing cells or cells at the resting state [51,52]. This is consistent with the fact that sporozoites obtained from mature oocysts are in the resting state and thus, the pyrimidine de novo biosynthesis pathway must be suppressed.

Because the oocysts sporulate under aerobic conditions and can use stored mannitol or amylopectin as carbon sources $[53,54]$, it is possible that the parasites need an active TCA cycle and oxidative phosphorylation for effective generation of ATP, since those internal carbon sources are limited. The mitochondrial complex II can function as succinate ubiquinone reductase (SQR) that catalyzes the oxidation of succinate and supplies electrons to the quinone pool, or as quinol fumarate reductase (QFR) by catalyzing the reverse reaction of SQR [6]. Complex II working as QFR becomes important under an environment of low oxygen, where the electron flux to oxygen through complexes III and IV are not functional. Such conditions cause an accumulation of respiratory quinols and, consequently, inhibition of all upstream ETC dehydrogenases. In order to re-oxidize quinols, complex II starts to function as QFR in a process called fumarate respiration, which is found in bacteria [55], helminthes [9,56], and also in some types of cancer cells living under a tumor microenvironment $[11,30,36,57,58]$. One of the important findings in the current study is that NADH started to re-oxidize by the addition of fumarate once the oxidation through complex III and IV was inhibited by cyanide. This fumarate-dependent NADH re-oxidation was completely inhibited by addition of malonate, a specific inhibitor of complex II. This means that E. tenella sporozoites has an intrinsic capacity of fumarate respiration in addition to classical oxygen respiration, which is supported by the presence of MK- $8\left(\mathrm{H}_{2}\right)$, a menaquinone specie (Figures S6 and S8). Such versatile mitochondrial respiration may become essential for adaptation to a hostile host intestinal environment. The presence of fumarate respiration in apicomplexan parasites has also been suggested for P. falciparum and $P$. berghei [59], which is consistent with our results.

We have previously reported that $P$. y. yoelii complex II was about 600 -fold less sensitive to atpenin A5 [39], the most potent complex II inhibitor described to date [24]. To our surprise, complex II from E. tenella was found to be even more insensitive to atpenin A5. In addition, other known inhibitors of complex II tested in this study, which were siccanin [25], carboxin [38], and flutolanil [7,27], did not inhibit E. tenella complex II, even at higher concentration of $50 \mu \mathrm{M}$ (Table 4). To our knowledge, E. tenella complex II is the most inhibitor-insensitive among this enzyme family reported so far.

Depending on the species, the composition of complex II subunits can be diverse. In general, complex II is composed by four subunits: Flavoprotein (Fp), iron-sulfur cluster (Ip), cytochrome $b$ large $(\mathrm{Cy} b \mathrm{~L})$, and small (CybS) subunits. However, complex II that physiologically functions as QFR is composed by three or four subunits and can be found in some bacteria and helminths, respectively [6]. Additionally, complex II formed by eight and 12 subunits was reported in plants [60] and trypanosomatid parasites, respectively [26,38]. Surprisingly, the E. tenella complex II analysed by $\mathrm{HrCNE}$ showed a single band of about $745 \mathrm{kDa}$ stained by the in-gel SDH-activity. In the following 2D-SDS-PAGE, a total of 15 bands stoichiometrically stained by CBB could be identified from SDH-activity stained band. The possibility of a trypanosomatid-type mega size complex II in E. tenella was ruled out because several complex III and IV subunits were identified in an SDH-stained band by nano-LC MS/MS analysis. Instead, our data suggest that the SDH-stained band has the potential to be a respiratory supercomplex formed by complexes II, III, and IV, which has never been reported. 


\section{Conclusions}

Our study provides the first evidence of an active and functional mitochondrial ETC in E. tenella parasites, which was only possible by the development of a new method for the isolation of mitochondria-rich fraction. Amongst the ETC enzymes, complex II showed unique biochemical features of the (i) ability to support fumarate respiration; (ii) being insensitive to practically all complex II inhibitors reported to date, and (iii) a possible formation of a respiratory supercomplex with complexes III and IV. Moreover, the differences in sensitivity to complex II inhibitors indicate that the development of E. tenella specific complex II inhibitor can be achieved and developed to a new generation of anticoccidian drugs with novel mechanism of action.

Supplementary Materials: The following are available online at http:/ /www.mdpi.com/2073-4425/10/1/29/s1, Table S1: Nano-LC MS/MS analysis of SDH activity stained band from HrCNE. Figures S1, S2, S3, S4 and S5: Alignment and percentage identity analysis of $\mathrm{NDH}-2, \mathrm{SDH} 1, \mathrm{SDH} 2, \mathrm{MQO}$ and G3PDH, respectively. Figures $\mathrm{S} 6$ and S7: Quinone content analysis from E. tenella sporozoites by HPLC-PDA and LC-MS, respectively.

Author Contributions: K.K. (Kiyoshi Kita), S.H., N.T., T.S. and K.S. conceived and designed the experiments; M.M., D.K.I., K.K. (Keisuke Komatsuya), T.H., K.H., J.Y. and K.S. performed the experiments; F.K. purified the oocysts; K.S. analyzed the nano-LC MS/MS data; M.M., D.K.I. and K.S. wrote the paper.

Funding: This work was supported in part by Infectious Disease Control from the Science and Technology Research Partnership for Sustainable Development (SATREPS, Nos. 10000284 to Ki.K. and 14425718 to D.K.I.); a Grant-in-aid for Scientific Research on Priority Areas 18073004 (to Ki.K.) and Creative Scientific Research Grant 18GS0314 (to Ki.K.) from the Japan Society for the Promotion of Science; a Grant-in-aid from the Program for the Promotion of Basic and Applied Research for Innovations in Bio-Oriented Industry (BRAIN) (No. 26020A to S.H. and Ki.K.); a Grant-in-aid for Scientific Research (B) 16K19114 to D.K.I and (C) 23570131 and 26234567 (to T.S.) from the Japanese Ministry of Education, Science, Culture, Sports and Technology (MEXT).

Conflicts of Interest: The authors declare no conflict of interest.

\section{References}

1. Naciri, P.M.; Brossier, F. Avian coccidiosis: Importance and research prospects. Bull. Acad. Vét France 2009, 162, 4. [CrossRef]

2. Li, G.Q.; Kanu, S.; Xiao, S.M.; Xiang, F.Y. Responses of chickens vaccinated with a live attenuated multi-valent ionophore-tolerant Eimeria vaccine. Vet. Parasitol. 2005, 129, 179-186. [CrossRef] [PubMed]

3. McDonald, V.; Shirley, M.W. The endogenous development of virulent strains and attenuated precocious lines of Eimeria tenella and E. necatrix. J. Parasitol. 1987, 73, 993-997. [CrossRef]

4. Kheysin, Y.M. Life Cycles of Coccidia of Domestic Animals; Willian Heinemann Medical Books Limited: London, UK, 1972.

5. Hermans, D.; Van Deun, K.; Martel, A.; Van Immerseel, F.; Messens, W.; Heyndrickx, M.; Haesebrouck, F.; Pasmans, F. Colonization factors of Campylobacter jejuni in the chicken gut. Vet. Res. 2011, 42. [CrossRef] [PubMed]

6. Harada, S.; Inaoka, D.K.; Ohmori, J.; Kita, K. Diversity of parasite complex II. Biochim. Biophys. Acta 2013, 1827, 658-667. [CrossRef] [PubMed]

7. Inaoka, D.K.; Shiba, T.; Sato, D.; Balogun, E.O.; Sasaki, T.; Nagahama, M.; Oda, M.; Matsuoka, S.; Ohmori, J.; Honma, T; et al. Structural insights into the molecular design of flutolanil derivatives targeted for fumarate respiration of parasite mitochondria. Int. J. Mol. Sci. 2015, 16, 15287-15308. [CrossRef] [PubMed]

8. Omura, S.; Miyadera, H.; Ui, H.; Shiomi, K.; Yamaguchi, Y.; Masuma, R.; Nagamitsu, T.; Takano, D.; Sunazuka, T.; Harder, A.; et al. An anthelmintic compound, nafuredin, shows selective inhibition of complex I in helminth mitochondria. Proc. Natl. Acad. Sci. USA 2001, 98, 60-62. [CrossRef]

9. Matsumoto, J.; Sakamoto, K.; Shinjyo, N.; Kido, Y.; Yamamoto, N.; Yagi, K.; Miyoshi, H.; Nonaka, N.; Katakura, K.; Kita, K.; et al. Anaerobic NADH-fumarate reductase system is predominant in the respiratory chain of Echinococcus multilocularis, providing a novel target for the chemotherapy of alveolar echinococcosis. Antimicrob. Agents Chemother. 2008, 52, 164-170. [CrossRef]

10. Iwata, F.; Shinjyo, N.; Amino, H.; Sakamoto, K.; Islam, M.K.; Tsuji, N.; Kita, K. Change of subunit composition of mitochondrial complex II (succinate-ubiquinone reductase/quinol-fumarate reductase) in Ascaris suum during the migration in the experimental host. Parasitol. Int. 2008, 57, 54-61. [CrossRef] 
11. Sakai, C.; Tomitsuka, E.; Esumi, H.; Harada, S.; Kita, K. Mitochondrial fumarate reductase as a target of chemotherapy: From parasites to cancer cells. Biochim. Biophys. Acta 2012, 1820, 643-651. [CrossRef]

12. Bulusu, V.; Jayaraman, V.; Balaram, H. Metabolic fate of fumarate, a side product of the purine salvage pathway in the intraerythrocytic stages of Plasmodium falciparum. J. Biol. Chem. 2011, 286, 9236-9245. [CrossRef]

13. Hartuti, E.D.; Inaoka, D.K.; Komatsuya, K.; Miyazaki, Y.; Miller, R.J.; Xinying, W.; Sadikin, M.; Prabandari, E.E.; Waluyo, D.; Kuroda, M.; et al. Biochemical studies of membrane bound Plasmodium falciparum mitochondrial L-malate:quinone oxidoreductase, a potential drug target. Biochim. Biophys. Acta 2018, 1859, 191-200. [CrossRef] [PubMed]

14. Niikura, M.; Komatsuya, K.; Inoue, S.I.; Matsuda, R.; Asahi, H.; Inaoka, D.K.; Kita, K.; Kobayashi, F. Suppression of experimental cerebral malaria by disruption of malate:quinone oxidoreductase. Malar. J. 2017, 16, 247. [CrossRef] [PubMed]

15. Tonhosolo, R.; Gabriel, H.B.; Matsumura, M.Y.; Cabral, F.J.; Yamamoto, M.M.; D'Alexandri, F.L.; Sussmann, R.A.C.; Belmonte, R.; Peres, V.J.; Crick, D.C.; et al. Intraerythrocytic stages of Plasmodium falciparum biosynthesize menaquinone. FEBS Lett. 2010, 584, 4761-4768. [CrossRef] [PubMed]

16. Painter, H.J.; Morrisey, J.M.; Mather, M.W.; Vaidya, A.B. Specific role of mitochondrial electron transport in blood-stage Plasmodium falciparum. Nature 2007, 446, 88-91. [CrossRef] [PubMed]

17. Scholtyseck, E. The Coccidia-Eimeria, Isospora, Toxoplasma and Related Genera; Hammond, D.M., Long, P.L., Eds.; University Park Press: Baltimore, MD, USA; London, UK, 1973; pp. 81-144.

18. Reid, A.J.; Blake, D.P.; Ansari, H.R.; Billington, K.; Browne, H.P.; Bryant, J.; Dunn, M.; Hung, S.S.; Kawahara, F.; Miranda-Saavedra, D.; et al. Genomic analysis of the causative agents of coccidiosis in domestic chickens. Genome Res. 2014, 24, 1676-1685. [CrossRef] [PubMed]

19. Matsubayashi, M.; Hatta, T.; Miyoshi, T.; Anisuzzaman; Sasai, K.; Shimura, K.; Isobe, T.; Kita, K.; Tsuji, N. High-throughput RNA sequencing profiles and transcriptional evidence of aerobic respiratory enzymes in sporulating oocysts and sporozoites of Eimeria tenella. Infect. Genet. Evol. 2013, 18, 269-276. [CrossRef] [PubMed]

20. Waterhouse, A.M.; Procter, J.B.; Martin, D.M.; Clamp, M.; Barton, G.J. Jalview Version 2-A multiple sequence alignment editor and analysis workbench. Bioinformatics 2009, 25, 1189-1191. [CrossRef]

21. Sheather, A.L. The detection of intestinal protozoa and mange parasites by a floatation technique. J. Comp. Pathol. Ther. 1923, 36, 10. [CrossRef]

22. Lowry, O.H.; Rosebrough, N.J.; Farr, A.L.; Randall, R.J. Protein measurement with the Folin phenol reagent. J. Biol. Chem. 1951, 193, 265-275.

23. Shimada, H.; Kodjabachian, D.; Ishida, M. Specific and rapid analysis of ubiquinones using Craven's reaction and HPLC with postcolumn derivatization. J. Lipid Res. 2007, 48, 2079-2085. [CrossRef] [PubMed]

24. Miyadera, H.; Shiomi, K.; Ui, H.; Yamaguchi, Y.; Masuma, R.; Tomoda, H.; Miyoshi, H.; Osanai, A.; Kita, K.; Omura, S. Atpenins, potent and specific inhibitors of mitochondrial complex II (succinate-ubiquinone oxidoreductase). Proc. Natl. Acad. Sci. USA 2003, 100, 473-477. [CrossRef] [PubMed]

25. Mogi, T.; Kawakami, T.; Arai, H.; Igarashi, Y.; Matsushita, K.; Mori, M.; Shiomi, K.; Omura, S.; Harada, S.; Kita, K. Siccanin rediscovered as a species-selective succinate dehydrogenase inhibitor. J. Biochem. 2009, 146, 383-387. [CrossRef] [PubMed]

26. Nihashi, N.; Inaoka, D.K.; Tsuge, C.; Balogun, E.O.; Osada, Y.; Goto, Y.; Matsumoto, Y.; Nara, T.; Mogi, T.; Harada, S.; et al. Siccanin is a novel selective inhibitor of trypanosomatid complex II (succinate-ubiquinone reductase) and a potent broad-spectrum anti-trypanosomatid drug candidate. In Kala Azar in South Asia: Current Status and Sustainable Challenges; Noiri, E., Jha, T.K., Eds.; Springer International Publishing: Cham, Switzerland, 2016.

27. Osanai, A.; Harada, S.; Sakamoto, K.; Shimizu, H.; Inaoka, D.K.; Kita, K. Crystallization of mitochondrial rhodoquinol-fumarate reductase from the parasitic nematode Ascaris suum with the specific inhibitor flutolanil. Acta Crystallogr. Sect. F Struct. Biol. Cryst. Commun. 2009, 65, 941-944. [CrossRef] [PubMed]

28. Von Jagow, G.; Ohnishi, T. The chromone inhibitor stigmatellin-Binding to the ubiquinol oxidation center at the C-side of the mitochondrial membrane. FEBS Lett. 1985, 185, 311-315. [CrossRef]

29. Yamashita, T.; Inaoka, D.K.; Shiba, T.; Oohashi, T.; Iwata, S.; Yagi, T.; Kosaka, H.; Miyoshi, H.; Harada, S.; Kita, K.; et al. Ubiquinone binding site of yeast NADH dehydrogenase revealed by structures binding novel competitive- and mixed-type inhibitors. Sci. Rep. 2018, 8, 2427. [CrossRef] [PubMed] 
30. Tomitsuka, E.; Kita, K.; Esumi, H. Regulation of succinate-ubiquinone reductase and fumarate reductase activities in human complex II by phosphorylation of its flavoprotein subunit. Proc. Jpn. Acad. Ser. B Phys. Biol. Sci. 2009, 85, 258-265. [CrossRef]

31. Mogi, T.; Kita, K. Diversity in mitochondrial metabolic pathways in parasitic protists Plasmodium and Cryptosporidium. Parasitol. Int. 2010, 59, 305-312. [CrossRef]

32. Madiraju, A.K.; Erion, D.M.; Rahimi, Y.; Zhang, X.M.; Braddock, D.T.; Albright, R.A.; Prigaro, B.J.; Wood, J.L.; Bhanot, S.; MacDonald, M.J.; et al. Metformin suppresses gluconeogenesis by inhibiting mitochondrial glycerophosphate dehydrogenase. Nature 2014, 510, 542-546. [CrossRef]

33. Sato, T.; Morita, A.; Mori, N.; Miura, S. The role of glycerol-3-phosphate dehydrogenase 1 in the progression of fatty liver after acute ethanol administration in mice. Biochem. Biophys. Res. Commun. 2014, 444, 525-530. [CrossRef]

34. Inaoka, D.K.; Iida, M.; Tabuchi, T.; Honma, T.; Lee, N.; Hashimoto, S.; Matsuoka, S.; Kuranaga, T.; Sato, K.; Shiba, T.; et al. The open form inducer approach for structure-based drug design. PLoS ONE 2016, 11, e0167078. [CrossRef] [PubMed]

35. Inaoka, D.K.; Sakamoto, K.; Shimizu, H.; Shiba, T.; Kurisu, G.; Nara, T.; Aoki, T.; Kita, K.; Harada, S. Structures of Trypanosoma cruzi dihydroorotate dehydrogenase complexed with substrates and products: Atomic resolution insights into mechanisms of dihydroorotate oxidation and fumarate reduction. Biochemistry 2008, 47, 10881-10891. [CrossRef] [PubMed]

36. Miyazaki, Y.; Inaoka, D.K.; Shiba, T.; Saimoto, H.; Sakura, T.; Amalia, E.; Kido, Y.; Sakai, C.; Nakamura, M.; Moore, A.L.; et al. Selective cytotoxicity of dihydroorotate dehydrogenase inhibitors to human cancer cells under hypoxia and nutrient-deprived conditions. Front. Pharmacol. 2018, 9, 997. [CrossRef] [PubMed]

37. Unden, G.; Bongaerts, J. Alternative respiratory pathways of Escherichia coli: Energetics and transcriptional regulation in response to electron acceptors. Biochim. Biophys. Acta 1997, 1320, 217-234. [CrossRef]

38. Morales, J.; Mogi, T.; Mineki, S.; Takashima, E.; Mineki, R.; Hirawake, H.; Sakamoto, K.; Omura, S.; Kita, K. Novel mitochondrial complex II isolated from Trypanosoma cruzi is composed of 12 peptides including a heterodimeric Ip subunit. J. Biol. Chem. 2009, 284, 7255-7263. [CrossRef] [PubMed]

39. Kawahara, K.; Mogi, T.; Tanaka, T.Q.; Hata, M.; Miyoshi, H.; Kita, K. Mitochondrial dehydrogenases in the aerobic respiratory chain of the rodent malaria parasite Plasmodium yoelii yoelii. J. Biochem. 2009, 145, $229-237$. [CrossRef] [PubMed]

40. Muhleip, A.W.; Joos, F.; Wigge, C.; Frangakis, A.S.; Kuhlbrandt, W.; Davies, K.M. Helical arrays of U-shaped ATP synthase dimers form tubular cristae in ciliate mitochondria. Proc. Natl. Acad. Sci. USA 2016, 113, 8442-8447. [CrossRef]

41. Krungkrai, J. The multiple roles of the mitochondrion of the malarial parasite. Parasitology 2004, 129, 511-524. [CrossRef]

42. Sturm, A.; Mollard, V.; Cozijnsen, A.; Goodman, C.D.; McFadden, G.I. Mitochondrial ATP synthase is dispensable in blood-stage Plasmodium berghei rodent malaria but essential in the mosquito phase. Proc. Natl. Acad. Sci. USA 2015, 112, 10216-10223. [CrossRef]

43. Lemgruber, L.; Lupetti, P. Crystalloid body, refractile body and virus-like particles in Apicomplexa: What is in there? Parasitology 2012, 139, 285-293. [CrossRef]

44. Wang, C.C.; Weppelman, R.M.; Lopez-Ramos, B. Isolation of amylopectin granules and identification of amylopectin phosphorylase in the oocysts of Eimeria tenella. J. Protozool. 1975, 22, 560-564. [CrossRef] [PubMed]

45. Komatsuya, K.; Hata, M.; Balogun, E.O.; Hikosaka, K.; Suzuki, S.; Takahashi, K.; Tanaka, T.; Nakajima, M.; Ogura, S.; Sato, S.; et al. Synergy of ferrous ion on 5-aminolevulinic acid-mediated growth inhibition of Plasmodium falciparum. J. Biochem. 2013, 154, 501-504. [CrossRef] [PubMed]

46. Siregar, J.E.; Kurisu, G.; Kobayashi, T.; Matsuzaki, M.; Sakamoto, K.; Mi-ichi, F.; Watanabe, Y.; Hirai, M.; Matsuoka, H.; Syafruddin, D.; et al. Direct evidence for the atovaquone action on the Plasmodium cytochrome $b c_{1}$ complex. Parasitol. Int. 2015, 64, 295-300. [CrossRef] [PubMed]

47. Takashima, E.; Takamiya, S.; Takeo, S.; Mi-ichi, F.; Amino, H.; Kita, K. Isolation of mitochondria from Plasmodium falciparum showing dihydroorotate dependent respiration. Parasitol. Int. 2001, 50, 273-278. [CrossRef] 
48. Hino, A.; Hirai, M.; Tanaka, T.Q.; Watanabe, Y.; Matsuoka, H.; Kita, K. Critical roles of the mitochondrial complex II in oocyst formation of rodent malaria parasite Plasmodium berghei. J. Biochem. 2012, 152, 259-268. [CrossRef] [PubMed]

49. Tanaka, T.Q.; Hirai, M.; Watanabe, Y.; Kita, K. Toward understanding the role of mitochondrial complex II in the intraerythrocytic stages of Plasmodium falciparum: Gene targeting of the Fp subunit. Parasitol. Int. 2012, 61, 726-728. [CrossRef]

50. Roberts, W.L.; Hammond, D.M. Ultrastructural and cytologic studies of the sporozoites of four Eimeria species. J. Protozool. 1970, 17, 76-86. [CrossRef]

51. Lane, A.N.; Fan, T.W. Regulation of mammalian nucleotide metabolism and biosynthesis. Nucleic Acids Res. 2015, 43, 2466-2485. [CrossRef]

52. Moreno-Morcillo, M.; Grande-Garcia, A.; Ruiz-Ramos, A.; Del Cano-Ochoa, F.; Boskovic, J.; Ramon-Maiques, S. Structural insight into the core of CAD, the multifunctional protein leading de novo pyrimidine biosynthesis. Structure 2017, 25, 912-923.e5. [CrossRef]

53. Ryley, J.F.; Bentley, M.; Manners, D.J.; Stark, J.R. Amylopectin, the storage polysaccharide of the Coccidia Eimeria brunetti and E. tenella. J. Parasitol. 1969, 55, 839-845. [CrossRef]

54. Schmatz, D.M. The mannitol cycle in Eimeria. Parasitology 1997, 114, S81-S89. [PubMed]

55. Van Hellemond, J.J.; Tielens, A.G. Expression and functional properties of fumarate reductase. Biochem. J. 1994, 304, 321-331. [CrossRef] [PubMed]

56. Kita, K.; Takamiya, S.; Furushima, R.; Ma, Y.C.; Suzuki, H.; Ozawa, T.; Oya, H. Electron-transfer complexes of Ascaris suum muscle mitochondria. III. Composition and fumarate reductase activity of complex II. Biochim. Biophys. Acta 1988, 935, 130-140. [CrossRef]

57. Tomitsuka, E.; Kita, K.; Esumi, H. The NADH-fumarate reductase system, a novel mitochondrial energy metabolism, is a new target for anticancer therapy in tumor microenvironments. Ann. N. Y. Acad. Sci. 2010, 1201, 44-49. [CrossRef] [PubMed]

58. Tomitsuka, E.; Kita, K.; Esumi, H. An anticancer agent, pyrvinium pamoate inhibits the NADH-fumarate reductase system-A unique mitochondrial energy metabolism in tumour microenvironments. J. Biochem. 2012, 152, 171-183. [CrossRef]

59. Fry, M.; Beesley, J.E. Mitochondria of mammalian Plasmodium spp. Parasitology 1991, 102, 17-26. [CrossRef]

60. Millar, A.H.; Eubel, H.; Jansch, L.; Kruft, V.; Heazlewood, J.L.; Braun, H.P. Mitochondrial cytochrome $c$ oxidase and succinate dehydrogenase complexes contain plant specific subunits. Plant Mol. Biol. 2004, 56, 77-90. [CrossRef]

(C) 2019 by the authors. Licensee MDPI, Basel, Switzerland. This article is an open access article distributed under the terms and conditions of the Creative Commons Attribution (CC BY) license (http://creativecommons.org/licenses/by/4.0/). 